\title{
Internet of Things based Driver Exhaustion Detection System using Distributed Sensor Network
}

\author{
Robbi Rahim \\ Sekolah Tinggi Ilmu Manajemen Sukma, Kota Medan, Indonesia. \\ robbirahim@ieee.org
}

Abstract-Accidents involving vehicles occur almost every day. Most of the accidents are caused due to exhaustion of driver's consciousness but this cause goes almost unreported due to the absence of systems to detect the fatigue. Techniques to measure driver fatigue can be invasive or non-invasive. All in all, driver-related information uncover a more grounded relationship to the driver's carefulness level than vehicle-related ones, which experience the ill effects of a bigger affectability to outer parameters, for example, street and traffic conditions. A measure of the amount of grip that the driver applies on the steering wheel is a more direct and non-invasive measure of his fatigue. An alcohol sensor when added to this system can further be used to check if the variation in grip is due to the effect of alcohol. A suitable algorithm is developed to confirm the occurrence of driver fatigue. The GSM and the GPS units attached to the system can be useful in notifying the control room in the event of driver fatigue. This project can be very useful in detecting fatigues in drivers associated with vehicle fleets using IoT.

Keywords- Vehicles, accidents, IoT

\section{Introduction}

Various technologies are available to make vehicular travel safer. The need for so much of technologies and research in this area is the fact that vehicular accidents are responsible for the maximum loss of lives each year. Car crashes have a major share in vehicular accidents. The crashes can occur due to a variety of reasons ranging from mechanical faults to human errors and much more. An important cause of car crashes that often goes unreported is Driver Fatigue. A driver who is too stressed out and hasn't rested is bound to lose his concentration and take more time to react to an emergency, thereby leading to an accident. This paper aims to detect driver fatigue on the basis of the variation of the amount of grip that he exerts on the steering wheel, thereby providing a non-invasive method of measuring driver fatigue.

An alcohol sensor is triggered once a variation in grip is detected to see if the driver is under the influence of alcohol. A suitable algorithm is developed to corroborate the variation in the grip to be due to driver fatigue. The project is designed to monitor the fatigue levels of drivers who are part of vehicle fleets. Vehicle fleets have now become a very common mode of transport in India. Once the sensors are triggered due to driver fatigue, the control room is notified with a text message that contains the information regarding the location of the vehicle. We believe that this system can be very successful in preventing accidents that are caused due to driver fatigue.

The amount of vehicles in Indian roads has risen exponentially in the past years. The amount of accidents has also increased in a drastic way. According to the "Global Status Report on Road safety", vehicular accidents cause 1, 30,000 deaths annually in India [1]. The National Crime Record Bureau states that 14 deaths occur due to road accidents every hour and it also estimates that the maximum number of accidents occur in the window between $3 \mathrm{pm}$ and $6 \mathrm{pm}$. The growth in the IT and other service sectors has caused an increase in the number of people who need to be ferried to their workplaces. Although many companies use buses for this purpose, a majority of the companies in the service sector whose employees work in shifts prefer using cabs to pick up and drop employees. Cabs have also begun to be widely used by other people due to their attractive pricing and ease of travel. As the cab drivers are forced to work in many shifts, they get tired and their fatigue level increases. This causes accidents. Also in the recent past many accidents have occurred involving drunk cab drivers.

Two or three attempts were taken to see the drowsiness of the driver by contemplating different parameters. Many existing systems executed vehicle-based examinations which mounted sensors for different bits of the vehicle $[3,4]$. The sensors are normally arranged on accelerating pedal and coordinating wheel, to review the power of the tiredness. The way toward finishing vehicle-based measures can be in addition eased back down into two classes. The assessment can be performed dependent upon two frameworks, expressly Steering Wheel Movement (SWM) and Standard Deviation of Lane Position (SDLP). So as to assess SWM, a point sensor is used to pick the driver's degree of laziness dependent on his overseeing model [5-7]. Obviously, SDLP finishes an outside camera which is 
utilized to pick if the vehicle is floating out of its way [8]. Regardless, assessments based vehicle-set up parameters remarkably depend with respect to the physical bits of the general condition and the driver himself. These elements fundamentally join the structure of the street, the sort of vehicle utilized and the driving instance of the driver which sway the exactness of the assessment [9]. In addition, such estimations are likewise used to see different wellsprings of auto crashes, for example, the tiredness dependent on liquor use. Therefore, vehicle-based estimations would not have the choice to see the reason behind the languor especially [10].

Adjacent to this, two or three ways of thinking finished mental measures to screen the drivers' depletion status which is executed by recording mental signs utilizing either electroencephalogram (EEG), electro-oculography (EOG), electromyography (EMG) or electrocardiography (ECG). One perfect circumstance of utilizing mental estimations is that the assessments dependent on such parameters can foresee lack of care with better precision since the mental signs can well address the passionate exercises of the cerebrum [2]. Regardless, such strategies are nosy since sensors are required to be put on the driver's body so as to amass the information. This may cause the driver to feel awkward and in addition include his idea from driving. Generally scarcely any current systems utilized energetic estimations which are composed through self-rating of the driver or through investigation [9]. In any case, such methods can incite difficult to miss result as the self-assessment methodology can caution the driver subliminally, diminishing the force of tiredness.

\section{II. EXISTING TECHNIQUES}

Today, it is very much perceived that driver weariness is a contributing component in countless street mishaps. In this way, creating smart frameworks for evaluating driver's carefulness level is a need. Such frameworks depend on the supposition that the event of exhaustion or sluggishness can be identified with quantifiable changes in driver's state and conduct. A critical point is along these lines the manner in which those progressions are uncovered, that is, the strategies utilized to recognize driver's weakness must be dependable, just as nonmeddling.

The methods to detect driver fatigue are of two types viz the ones using the signals from the driver and could be invasive or non-invasive and the ones which depend on the signals from the vehicle. The latter approach requires a large number of sensors to monitor multiple parameters in the vehicle like speed, road surface condition and time of the day. The approach is computationally intensive and costly. Frameworks which use signals from the driver to quantify exhaustion incorporate physiological parameters, for example, electroencephalogram, electrocardiogram, electro-myogram and skin conductivity, whose measure for the most part expects cathodes to be applied to the driver. These systems are effective and are direct evidences of driver fatigue, but these systems are intrusive and can be a hindrance to the driver.

Technologies which claim to detect driver fatigue by observing the brain waves non-intrusively are still in their elementary stages and their accuracy is questionable. Other driver-related signs are eye development, head position, and outward appearance, which can be gained utilizing cameras and PC vision [2]. Such techniques are computationally intensive and require complex algorithms to be implemented. Further the data can be erroneous due to the poor condition of the roads in India. In a market where the cost of the cars is quite less like in India, use of such computationally intensive systems increases the cost. A promising technique is what utilizes the hold power that a driver applies to the guiding wheel in the driver's hypocautiousness location frameworks [3] [4] [5]. Notice that the adequacy of such frameworks can altogether be improved through the combination of various types of information utilizing an appropriate calculation. Albeit a few promising philosophies for driver exhaustion recognition have been proposed in writing, much work despite everything should be done in the examination of compelling answers for their down to earth execution. The current procedure is appeared in figure 1.

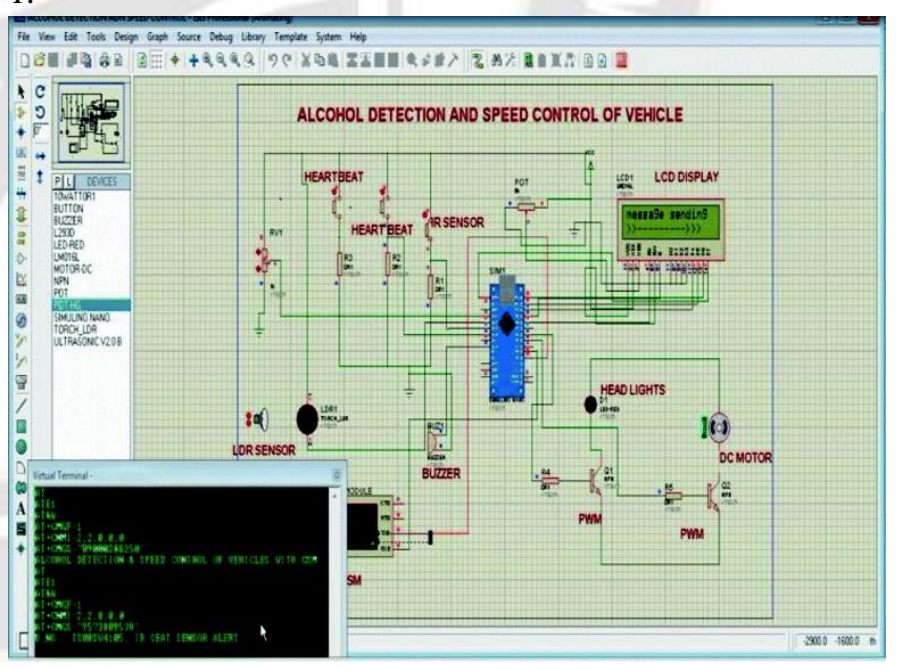

Fig 1: Structure of the existing system

Some momentum led to model-set progression following the eye state and the head position of the driver. This method proposes high precision rate with acceptably low blunders and fake alerts for individuals of different ethnicity and sexual bearing. Regardless, this has an affront of higher figuring limit need and the reaction of being delicate to mayhem. Intimating the starting late demonstrated issues [9]; we have proposed a 
method, which depends upon the social estimations, wherein the eye end degree is utilized as the information parameter for perceiving driver's drowsiness. Strategy depended on social estimations fuse checking the eye flickering model, yawning, eye end, facial unforeseen developments and head present by strategies for an outside camera. Besides, as the framework is composed contemplating the immediate parameters, it fills in as a non-meddling method of picking the driver's lack of care as it doesn't require any conditions of sensors on the driver's body and along these lines doesn't burst in on him while driving. In our proposed structure, the essence of the driver was persistently recorded so as to perceive the eye enhancements utilizing a Raspberry Pi camera.

To adequately get the face, the Pi camera is mounted on the vehicle dashboard and is gotten around $20 \mathrm{~cm}$ far from the driver's face. This Pi camera is connected with the Raspberry Pi with the assistance of a flexible association and the Raspberry Pi itself can be put any place inside the vehicle, out the human visual insight. From the beginning, the disclosure of facial spots of intrigue was performed utilizing the Haar Cascade classifier. Right when the different zones of the face were perceived, the eye regions were evacuated to check the eye end degree. On the off chance that the eye end degree of the driver separate subject to what is viewed as the standard degree of a person in a typical express, the jumper is in a concise second startled with the assistance of the ringer. Moreover, to guarantee the driver is taking real checks to not nod off, the proprietor of the vehicle is told too through an email which will be sent in this manner if the driver is seen as resting off in excess of a few times. The whole structure was united utilizing a Raspberry Pi and a Pi camera was utilized for following the eye headways.

\section{Proposed Methodology}

The distributed network of sensors include the grip sensor that forms the primary tool for detecting the driver's fatigue and the alcohol sensor which is used to find if the behaviour of the drive is due to the effect of alcohol. It is the behaviour of some drivers to occasionally loosen the grip on the steering wheel occasionally, in order to compensate for this and to avoid a situation in which such behaviour is considered as driver fatigue, we analyze the behaviour of the driver, his general ways of holding on to the steering and we use this value to calibrate the system. Once we have a variation in the grip above or below this calibrated value, the system triggers the alarm.

When a driver is under fatigue he tends to lose his grip on the vehicle, this may also arise if the driver is under the influence of alcohol so the alcohol sensor is activated. If the alcohol sensor is also triggered, then the vehicle is immediately stopped using the relay driver.
The controller unit in the vehicle is the ARM7 LPC2129 microcontroller which operates using 32 bits. The control unit receives the data from the sensors and in the event of the sensors getting triggered, the control unit first triggers the alarm system. Further the control unit instructs the relay driver to stop the vehicle. In this project the stopping is implemented by stopping the dc motor. Further the controller calls for the position information from the GPS unit and then sends the position information to the number specified (generally the control room's number). In the opposite route, the controller unit can also receive instructions from the control room to stop the vehicle if an emergency occurs.

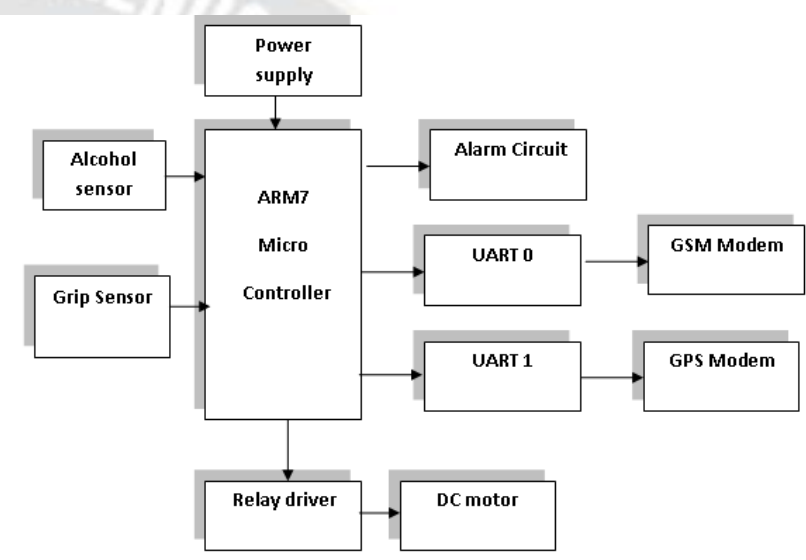

Fig 2: Structure of the proposed system

The system consists of the following units:
a) The sensors
b) The control unit
c) Communication and positioning units
d) Vehicle control units
e) IoT based Server

The algorithm is shown in figure 3. Initially the grip sensor is calibrated to the nominal values of grip that the driver applies on the steering wheel. 


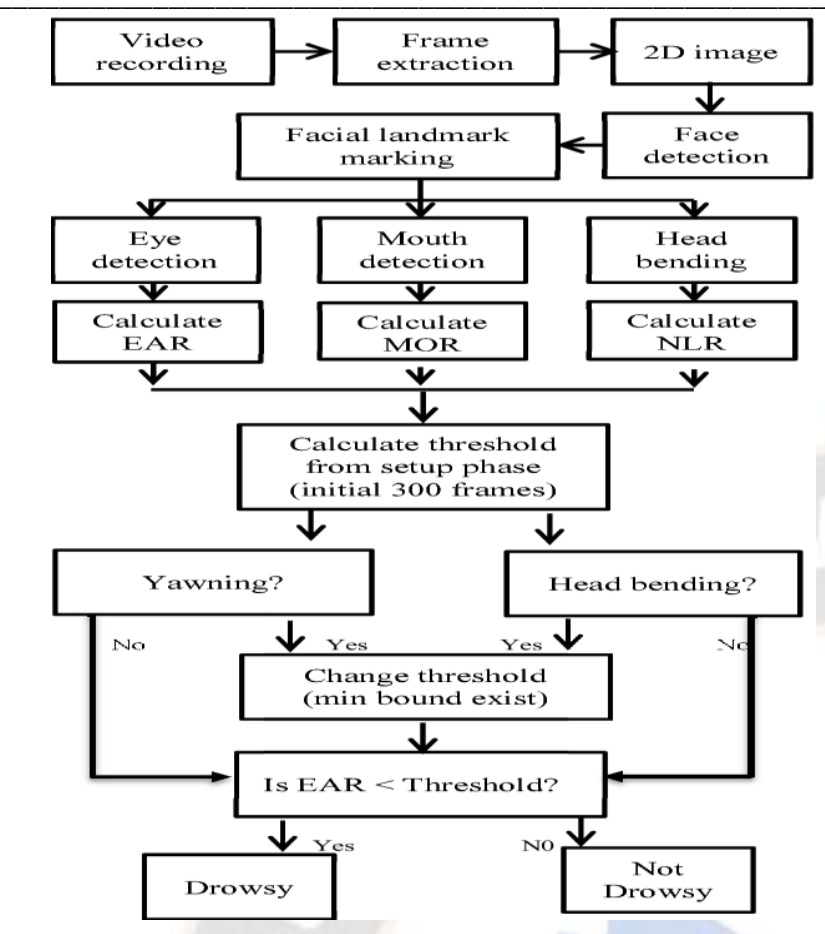

Fig 3: Algorithm for Driver Exhaustion Detection System

\section{Simulation Results}

The communication and positioning units in the system are used to accurately determine the position of the vehicle so that the control room can take necessary action. The system transmits information to the control room as per the instructions of the controller unit.

The information sent to the control room has the following format,

a) Warning...Alcohol Detected :: Location of the vehicle: $98.3 \mathrm{E} 107.4 \mathrm{~N}$

b) Warning...Driver fatigue :: Location of the vehicle: $84.5 \mathrm{~W} 135 \mathrm{~N}$

The vehicle control unit includes the relay driver that is used to stop the vehicle on the instructions of the controller unit. Here the effect is emulated by controlling a dc motor. The alarm system is sounded once the sensors are triggered. The next stage after the alarm is detected is the stopping of the vehicle as shown in figure 4 .

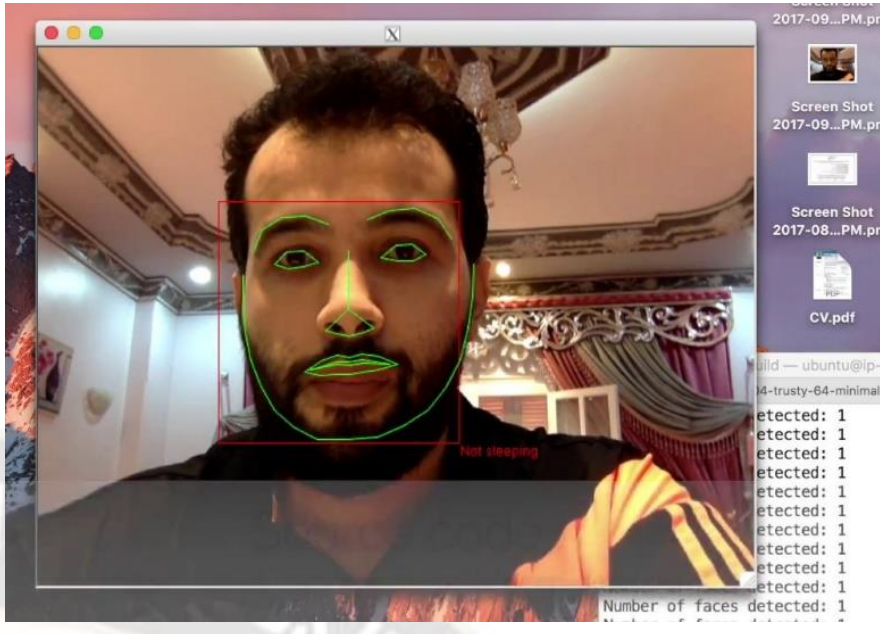

Fig 4: Simulation result for Driver Exhaustion Detection

$$
\text { System }
$$

The proposed system is a non-invasive method of detecting driver fatigue and is shown in figure 4 . The driver need not wear any sensor system on his body thereby reducing any disturbance caused to the driver. An alcohol sensor is used to find if the variation in pressure is due to the influence of alcohol. In addition to preventing drunk driving, the system also provides a method of further narrowing down the possible reason for fatigue. The control unit in this project is implemented using the ARM7 LPC2129 which is a 32 bit microcontroller offering superior performance than other microcontrollers.

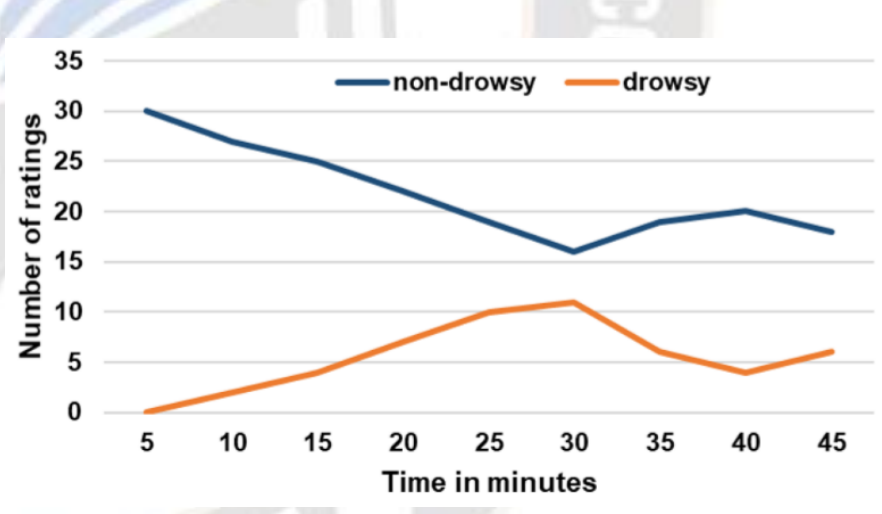

Fig 5: Comparison chart showing drowsy and non-drowsy moments

The use of the GSM and GPS systems ensure that the location of the vehicle can be accurately determined and the control room once notified can take appropriate action like sending medical help or arranging an alternate vehicle. The system can be very much useful for companies operating large vehicle fleets including trucks and cars as the GSM and GPS systems have a wide reach. The planning dataset used to set up the photos was not appropriate for a wide scope of environmental conditions. It involved pictures that were taken interestingly in daylight. Regardless, while finishing the assessment around night time, it was seen that the figuring couldn't distinguish the facial places of interest from the video housings of the driver. 
Along these lines, the system couldn't register the EAR estimation of the driver's eye. This brought repressions for our system as it could simply fill its need during the light. This is shown in figure 6.

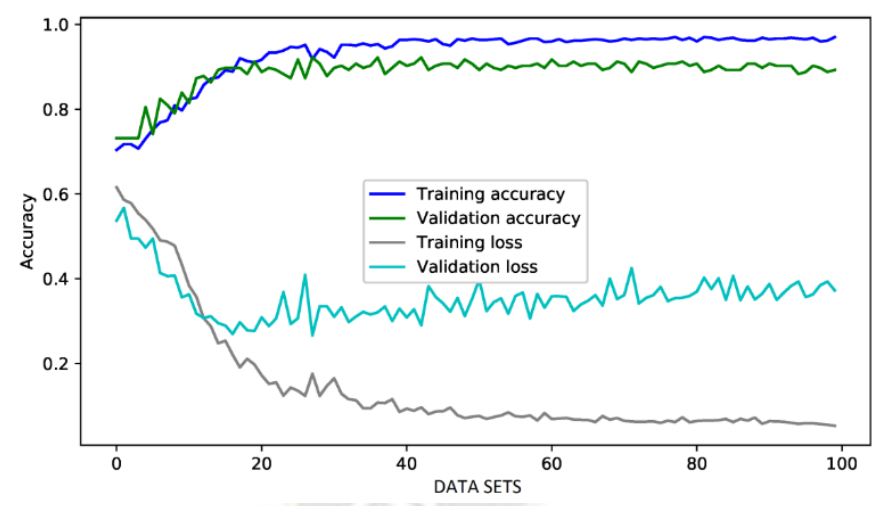

Fig 6: Comparison chart showing dataset and their accuracy

The EAR picks how far the eyelids of each eye are confined from one another. Notwithstanding, when an individual flickers, the EAR decreases basically, pushing toward zero. The common scope of single eye squint of every individual goes around from 100 to $400 \mathrm{~ms}$ as showed by [4]. From these estimations, it will when all is said in done be found that the term of eye end must be a higher priority than $400 \mathrm{~ms}$ for an individual who is perceived with languor. In our work, four edges were considered to address this $400 \mathrm{~ms}$, showing that four powerful edges with an eye point degree under 0.25 must happen all together for a squint to be enlisted. In this way, it is conceivable to see the eye end structure between the eye squint and sluggish eyes.

\section{Conclusion}

This research article can be very much useful if it is implemented in a proper manner. The advantage of this system is that it involves the latest available technologies in detecting and issuing appropriate warning signals way before the effects of driver fatigue lead to disastrous events. The location information can also be interfaced with mapping software like Google Maps to specify the exact position of the vehicle. The control room can then take appropriate action like sending in alternative vehicles or arranging for medical help for the drivers. The proposed method shows better performance compared to conventional approaches.

\section{References}

[1] Tianyi Qin, Drivers drowsiness detection in embedded system, IEEE International Conference on Vehicular Electronics and Safety, 2007. ICVES

[2] Baronti et al, "Distributed sensor for steering wheel grip force measurement in driver fatigue detection",
Europe Conference \& Exhibition in Design, Automation \& Test, 2009, pp-894-897

[3] T. Pilutti and A. Ulsoy, "Identification of driver state for lane-keeping tasks," IEEE Trans. Syst., Man, Cybern. A, vol. 29, no. 5, pp. 486-502, Sep 1999.

[4] T. C. Chieh, M. M. Mustafa, A. Hussain, E. Zahedi, and B. Y. Majlis, "Driver fatigue detection using steering grip force," in Proc. Student Conf. on Research and Development (SCORED 2003), Aug. 2003, pp. 45-48.

[5] C. C. Liu, S. G. Hosking, and M. G. Lenné, "Predicting driver drowsiness using vehicle measures: Recent insights and future challenges," Journal of Safety Research, vol. 40, no. 4, pp. 239-245, 2009.

[6] S. Otmani, T. Pebayle, J. Roge, and A. Muzet, "Effect of driving duration and partial sleep deprivation on subsequent alertness and performance of car drivers," Physiology \& behavior, vol. 84, no. 5, pp. 715-724, 2005.

[7] S. H. Fairclough and R. Graham, "Impairment of driving performance caused by sleep deprivation or alcohol: a comparative study," Human factors, vol. 41, no. 1, pp. 118-128, 1999.

[8] R. Simons, M. Martens, J. Ramaekers, A. Krul, I. Klöpping-Ketelaars, and G. Skopp, "Effects of dexamphetamine with and without alcohol on simulated driving," Psychopharmacology, vol. 222, no. 3, pp. 391-399, 2012.

[9] X. Ma, L. Chau and K. Yap, "Depth video-based twostream convolutional neural networks for driver fatigue detection," 2017 International Conference on Orange Technologies (ICOT), Singapore, 2017, pp. 155-158, doi: 10.1109/ICOT.2017.8336111.

[10] F. Zhang and F. Dai, "Research and Application of Fatigue Driving Monitoring and Emergency Treatment," 2017 International Conference on Computer Technology, Electronics and Communication (ICCTEC), Dalian, China, 2017, pp. 1072-1075, doi: 10.1109/ICCTEC.2017.00235. 